\title{
0 impacto da visão monocular congênita versus adquirida na qualidade de visão autorrelatada
}

\author{
Impact of congenital versus acquired monocular vision as self-reported vision
}

Marcelo Caram Ribeiro Fernandes ${ }^{1}$, Guilherme Pellizzer Marin ${ }^{1}$, Antonio Brunno Vieira Nepomucen ${ }^{1}$, Fábio Marques do Nascimento ${ }^{1}$, Eduardo MELANI ROCHA ${ }^{1}$

\section{RESUMO}

Objetivos: Quando a visão de um olho está preservada (visão monocular) e há alto risco, baixo prognóstico e/ou recursos limitados para a cirurgia do olho contralateral, não é claro se o beneficio da binocularidade supera o da reorientação para visão monocular. O objetivo é quantificar o impacto da qualidade de visão referida entre a condição binocular e monocular e, nesse último caso, entre congênita e adquirida.

Métodos: Pacientes com acuidade visual ( $A V$ ) com $A V>0,5$ em cada olho foram submetidos ao questionário estruturado de 14 perguntas (VF-14), onde a pontuação de 0 a 100 indica o nível de satisfação do paciente com sua visão, variando de baixo a alto respectivamente. Dados epidemiológicos e pontuações dos quatro grupos foram registrados e submetidos à análise estatística. Resultados: A entrevista pelo VF-14 com 56 indivíduos revelou que a pontuação mais alta foi similar entre os controles e os portadores de visão monocular congênita, e níveis intermediários e baixos foram obtidos por indivíduos com visão monocular adquirida e cegos bilaterais, respectivamente $(p<0,001)$. As atividades mais difíceis para os indivíduos com visão monocular adquirida foram identificar letras pequenas, reconhecer pessoas, distinguir sinais de trânsito e assistir TV.

Conclusão: O estudo confirmou que a perda da visão tem impacto desfavorável no desempenho referido das atividades sendo maior na visão monocular adquirida do que na congênita. Os dados sugerem que medidas de reabilitação devem ser consideradas para melhorar a qualidade da visão em doenças intratáveis ou de alto risco ou de baixo prognóstico.

Descritores: Visão monocular; Baixa visão; Cegueira/epidemiologia; Acuidade visual; Qualidade de vida; Visão binocular; Questionários

\begin{abstract}
Purpose: When the vision in one eye is preserved (monocular vision) and there is high risk, low prognosis and/or limited resources to the fellow eye surgery, it is unclear if the benefit of binocularity outweighs the reorientation formonocular vision. The goal is to quantify the impact of the quality of vision of both binocular and monocular condition, and in this latter case, between congenital and acquired.

Methods: Patients with visualacuity (VA)>0.5 in eacheye underwentastructured questionnaire of 14 questions (VF-14), which the score 0-100 indicates the level of patient satisfaction with their vision, ranging from low to high respectively. Epidemiological data and scores of the four groups were recorded and analyzed statistically.

Results: The interview by the VF-14 with 56 subjects revealed that the highest score was similar between controls and patients with congenital monocular vision, and low and intermediate levels were obtained by individuals with acquired monocular vision and bilaterally blind, respectively $(p<0.001)$. The more difficult activities for individuals with acquired monocular vision were to identify small print, recognize people, to distinguish traffic lights and watch TV. Conclusion: The study confirmed that the vision loss has an adverse impact on the performance of such activities being higher in congenital than in acquired monocular vision. The data suggest that rehabilitation measures should be considered to improve the quality of vision in intractable diseases, high risk or low prognosis.
\end{abstract}

Keywords: Vision, monocular; Vision, low; Blindness/epidemiology; Visualacuity; Quality of life; Vision, binocular; Questionnaires

\section{INTRODUÇÃO}

A s causas mais frequentes de baixa de visão potencialmente reversíveis com cirurgia estão relacionadas à catarata e opacidade de córnea ${ }^{(1-2)}$. Atualmente a cirurgia mais realizada em todo o mundo é a cirurgia de catarata ${ }^{(3)}$. Da mesma forma o transplante sólido mais realizado é o de córnea ${ }^{(4)}$. Por outro lado, estima-se que entre 5 e $9 \%$ da população tenha baixa de visão ou cegueira monocular ${ }^{(1-2)}$.

Trabalho realizado no Departamento de Oftalmologia, Otorrinolaringologia e Cirurgia de Cabeça e Pescoço, Faculdade de Medicina de Ribeirão Preto, Universidade de São Paulo Ribeirão Preto (SP), Brasil.

${ }^{1}$ Médico, Departamento de Oftalmologia, Otorrinolaringologia e Cirurgia de Cabeça e Pescoço, Faculdade de Medicina de Ribeirão Preto - Universidade de São Paulo - USP Ribeirão Preto (SP), Brasil.

Endereço para correspondência: Eduardo Melani Rocha. Departamento de Oftalmologia, Otorrinolaringologia e Cirurgia de Cabeça e Pescoço, Faculdade de Medicina de Ribeirão Preto - Universidade de São Paulo. Av. Bandeirantes, 3.900 - Ribeirão Preto (SP)

CEP 14049-900 - E-mail: emrocha@fmrp.usp.br

Apoio financeiro: FAPEA, CNPq

Recebido para publicação em 17.06.2010

Aprovação em 15.11.2010
Os diagnósticos relacionados e também a disponibilidade de recursos financeiros e técnicos variam em diferentes regiões do mundo(5-6). Da mesma forma, os riscos envolvidos e o prognóstico cirúrgico variam com indivíduos e as combinações de doenças ${ }^{(7)}$. Contudo, os prejuízos individuais e coletivos de baixa de visão, principalmente monocular, versus os benefícios conquistados com sua reabilitação, nunca foram sistematicamente avaliados.

A cirurgia no segundo olho em caso de doenças bilaterais ou a reabilitação de um olho acometido por um problema óptico em caso de olho contralateral sadio não são consensos e permanecem objeto de estudo ${ }^{(8-9)}$. A decisão passa por diversos aspectos, entre eles, acesso ao tratamento, disponibilidade de recursos, potenciais riscos de intervenção e sobrevida do resultado alcançado ${ }^{(10-11)}$.

Os cirurgiões de catarata que advogam a cirurgia bilateral simultânea justificam sua conduta, alegando que o sistema visual humano é binocular por natureza e que a percepção de profundidade monocular, usando paralaxe ou estratégias aprendidas não são suficientes na percepção dos pacientes ${ }^{(12-13)}$.

A recente discussão no Brasil de considerar incapaz o paciente com visão monocular e, portanto, sujeito a benefícios previdenciários, somado ao envelhecimento da população e à 
perda de visão monocular por trauma, tornam o tema de interesse comunitário, além do interesse clínico em prever o real beneficio do tratamento ao paciente ${ }^{(14)}$. Estudos anteriores com esse objetivo avaliaram pacientes antes e depois do tratamento, sendo, em sua maioria, amostras não aleatórias, não-mascaradas ou sem especial atenção ao potencial de adaptação à visão monocular, portanto limitações e vieses, favorecendo a intervenção, não são descartados ${ }^{(15-17)}$.

Pelas razões apresentadas, o objetivo desse estudo foi avaliar o impacto da visão monocular na qualidade de visão referida por pessoas com cegueira monocular, congênita e adquirida, no desempenho de suas atividades diárias, comparado a pacientes com visão normal e cegos binoculares, usando o questionário "Visual Function-14" (VF-14)"(18).

\section{MÉTODOS}

Após a avaliação e aprovação da comissão de ética em pesquisa e obtenção do consentimento esclarecido dos pacientes, foram entrevistados 56 pacientes do Hospital das Clínicas da Faculdade de Medicina de Ribeirão Preto - USP, distribuídos nos grupos: cego binocular (cuja visão reduzida, seja congênita ou adquirida, não oferece apoio satisfatório na execução das atividades questionadas no VF-14), monocular congênito (cujo aprendizado de atividades se fez com a visão de um único olho), monocular adquirido (cujas atividades foram aprendidas e executadas com a visão binocular, até um período recente, não inferior a 3 anos) e controle com visão normal.
O questionário VF-14 consiste de 14 perguntas que correspondem a atividades às quais o entrevistado respondeu quanto a sua dificuldade em realizá-las. A resposta foi quantificada (nenhuma dificuldade, pouca, moderada, muita ou incapaz). 0 item foi excluído do cálculo de pontuação quando o indivíduo não realizava a atividade ou a impossibilidade de realizá-la era por outra razão que não o déficit visual. Os resultados foram obtidos em valores de 0 (incapaz de realizar qualquer atividade) a 100 (realiza todas as atividades com muita facilidade), em intervalos de 20. Dessa forma, pontuações mais altas estão relacionadas a um melhor desempenho visual.

Foi também perguntado ao entrevistado sobre a intensidade do seu problema (nenhum, pouco, moderado, muito) e quanto a sua satisfação com a visão (péssimo, muito insatisfeito, insatisfeito, satisfeito, muito satisfeito)

Os dados analisados foram aspectos epidemiológicos dos grupos, número de pontos obtidos no questionário VF-14 (Anexo 1), acuidade visual em cada olho e do tempo de perda da visão nos casos com cegueira mono e binocular.

A inclusão se limitou a pacientes maiores de 18 anos, recrutados no Ambulatório de Oftalmologia e a controles, entre os acompanhantes saudáveis ou membros da equipe do hospital. A definição do problema como congênito ou adquirido foi feita pela história e dados do prontuário médico. A definição de cegueira foi a adotada pela Organização Mundial de Saúde, de acuidade visual $<0,1$ ou 20/200.

Foram excluídos pacientes com outras doenças crônicas que levassem a limitação da qualidade de vida (cognitiva, motora,

\section{Anexo 1. Questionário VF-14}

Nome:

Registro:

Data de nascimento:

Diagnóstico:

AV:

Você tem alguma dificuldade, mesmo com óculos para:

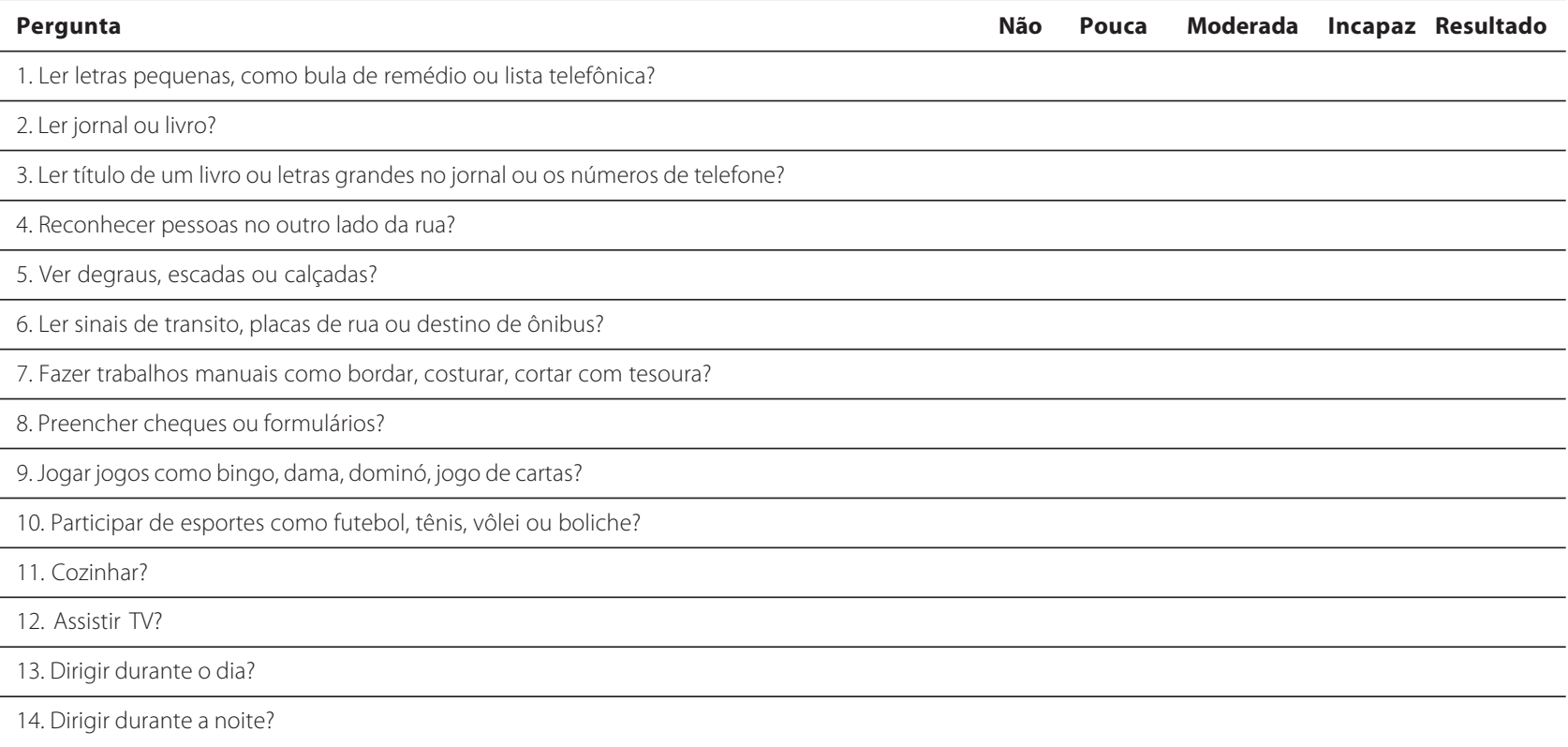

\begin{tabular}{l}
\hline Perguntas extras \\
\hline 1. Você tem algum problema com a visão (nenhum, pouco, moderado, muito, muito mesmo) \\
\hline 2. Qual e o grau de satisfação com a visão (péssimo, muito insatisfeito, insatisfeito, satisfeito, muito satisfeito) \\
\hline OBS:
\end{tabular}


ou uso de duas ou mais medicações sistêmicas) ou que pudessem indiretamente influenciar a realização das tarefas elencadas e portanto as respostas. Foram também excluídos pacientes sem percepção luminosa bilateral ou perda de visão há menos de três anos.

Para análise estatística, a acuidade visual obtida com a melhor correção foi convertida para a escala logMAR. Acuidade visual de conta dedos foi atribuída 0,02, de movimento de mãos, 0,01 e de percepção luminosa 0,005 na escala decimal e a partir daí convertidas também para logMAR. As médias e desvio-padrão foram calculados levando em conta a visão do melhor olho, assumindo que a visão binocular seria próxima da visão desse olho. Para melhor entendimento de leitores não familiarizados com a escala logMAR, as médias foram também expressas na escala decimal.

Foi feita a estatística descritiva dos grupos para comparação. A análise estatística dos pontos foi feita comparando as médias das notas obtidas no VF-14 entre os grupos por ANOVA, seguido de teste post-hoc de Bonferroni, e nos casos com perda adquirida, por correlação da nota com o tempo de acometimento. A mesma estatística por ANOVA foi usada na comparação da melhor visão entre os quatro grupos.

\section{RESULTADOS}

Os pacientes dos diferentes grupos tiveram distribuição etária e por sexos registrados, sendo o grupo de cegueira monocular adquirida mais velho e com predominância masculina (Tabela 1). A acuidade visual média foi similar entre controles e os dois grupos de cego monocular, e obviamente inferior no grupo de cegos binoculares ( $p<0,01$, ANOVA e teste post hoc Bonferroni) (Tabela 1).

O tempo médio de perda da visão entre os indivíduos com visão monocular adquirida foi de 5,3 \pm 1,0 anos e nos cegos binoculares foi de 12,7 $\pm 4,0$ anos. As atividades profissionais foram variadas dentro e entre os grupos. O tempo de escolaridade foi similar entre os grupos, com 6,8 $\pm 3,0$ nos controles, 5,5 $\pm 3,5$ nos indivíduos com visão monocular congênita, 6,6 \pm 3,7 nos indivíduos com visão monocular adquirida e 6,6 $\pm 2,9$ nos cegos binoculares.
As principais doenças causadoras de baixa de visão, nesse levantamento foram doenças da córnea e da retina, seguidas por ambliopia e glaucoma (Tabela 2).

Em relação à pontuação no VF-14, os grupos tiveram pontuações marcadamente diferentes, exceto o grupo monocular congênito, que referiu pontuação semelhante ao grupo controle. As seguintes médias e desvio-padrão foram observadas: $95,5 \pm 4,0$ para o grupo controle, $96,4 \pm 3,8$ para o grupo de cegos monoculares congênitos, $64,1 \pm 20,3$ para o grupo com cegueira monocular adquirida e 15,4 \pm 9,5 para o grupo de cegos binoculares ( $p=0,0005$, com diferença entre os grupos confirmada pelo teste post hoc de Bonferroni) (Figura 1).

A satisfação referida com a visão e a existência de problemas com a visão, ambas aferidas também pelo VF-14, revelou boa correlação entre esses parâmetros e entre eles e a pontuação total nas questões do VF-14. Entre os controles, todos referiram satisfação e nenhum referiu problemas, no grupo de visão monocular congênito, os mesmos 13 referiram satisfação e ausência de problemas e 1 referiu insatisfação e problema com a visão e entre os cegos binoculares, apenas 1 entre 14 indivíduos, referiu satisfação e ausência de problemas. O grupo com cegueira monocular adquirida apresentou também, como na pontuação do VF-14, maior variabilidade interna ao referir satisfação com a visão, com apenas 6, entre 14, afirmando satisfação e todos os 14 referiram graus variados de problemas com a visão.

Entre os cegos binoculares, algumas respostas indicaram relativa facilidade para realizar tarefas como cozinhar e praticar esporte, mas na grande maioria dos casos, o questionário revelou dificuldade moderada ou grande na maioria das atividades. O grupo com visão monocular congênita teve 2 respostas de relativa dificuldade para letras pequenas e atividades manuais, no restante as respostas foram máximas ou próximas disso em todos os itens aplicáveis. No grupo de visão monocular adquirida, a distribuição de respostas foi heterogênea, porém os itens que tiveram maior frequência de dificuldade foram as relacionadas a letras pequenas, reconhecimento de pessoas à distância, sinais de trânsito e ver TV.

As perguntas que tiveram maior frequência de respostas "Não se aplica" (entre 29 e 50\%) e, portanto, foram desconsi-

Tabela 1. Dados epidemiológicos dos pacientes entrevistados para qualidade de visão pela condição de visão monocular e binocular (idade e acuidade visual logMAR expressos em média \pm desvio padrão). A acuidade visual média foi também expressa na escala decimal

\begin{tabular}{lcccc}
\hline Grupo & Idade & Sexo F: M & Acuidade visual logMAR & Acuidade visual escala decimal \\
\hline Controle & $38,3 \pm 14,2$ & $8: 7$ & $0,0 \pm 0,0$ & 1,0 \\
Cego monocular adquirido & $59,5 \pm 20,2^{*}$ & $3: 10$ & $0,16 \pm 0,15$ & 0,7 \\
Cego monocular congênito & $40,8 \pm 16,6$ & $7: 7$ & $0,02 \pm 0,04$ & 0,9 \\
Cego binocular & $55,0 \pm 18,4$ & $7: 8$ & $1,30 \pm 0,40^{*}$ & $0,02^{*}$ \\
\hline
\end{tabular}

${ }^{*}=p<0,05$ (ANOVA, post hoc Bonferroni)

Tabela 2. Causas de baixa de visão entre pacientes entrevistados para qualidade de visão pela condição de visão monocular e binocular

\begin{tabular}{lccc}
\hline & Controle & Cego monocular adquirido & Cego monocular congênito \\
\hline Ambliopia & 0 & 0 & 8 \\
Glaucoma & 0 & 0 & 0 \\
Opacidade de córnea & 0 & 7 & 6 \\
Alteração de retina & 0 & 4 & 0 \\
Catarata & 0 & 1 & 5 \\
Neuropatia óptica & 0 & 1 & 0 \\
Total & 15 & 13 & 0 \\
\hline
\end{tabular}




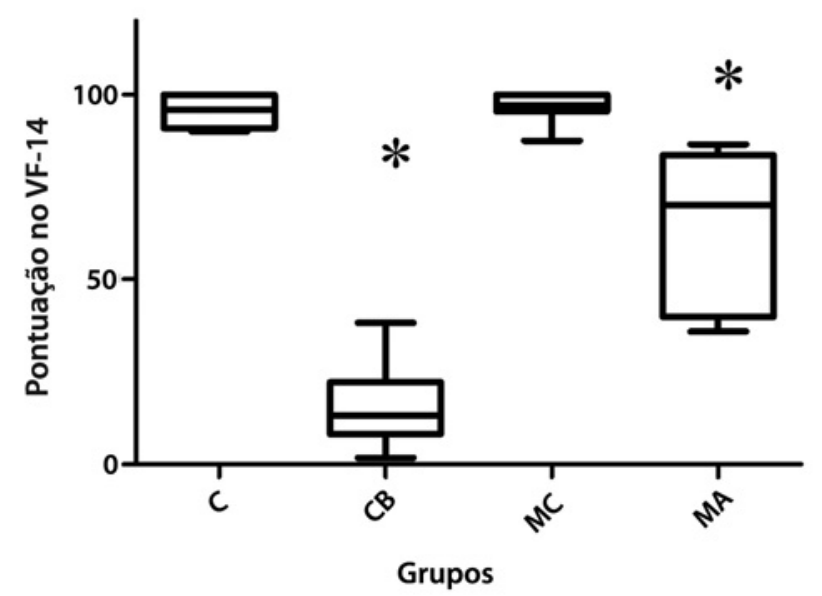

Figura 1.Pontuação no questionário de qualidade de visão VF-14, entre pacientes entrevistados para qualidade de visão em indivíduos controles (C), com cegueira binocular (CB), portadores de visão monocular congênita (MC) e visão monocular adquirida (MA).

deradas no cálculo da pontuação desses indivíduos foram as relacionadas a jogos, esportes, condução de automóvel de dia e a noite.

\section{DISCUSSÃO}

O presente estudo procurou avaliar o impacto da visão monocular no desempenho de atividades que dependem da visão em indivíduos normais, cegos monoculares congênitos, monoculares adquiridos e cegos binoculares pelo questionário VF-14.

A observação de que a visão monocular congênita tem menor efeito sobre a qualidade da visão do que a adquirida sugere que a execução das tarefas que exigem participação da visão, pode ser aprendida. O estudo mostrou que visão de perto e reconhecimento de faces, sinais e TV são os maiores obstáculos em pessoas com visão monocular.

Isso tem aplicação prática nas decisões relacionadas a trauma monocular, cujo tratamento cirúrgico para reverter as opacidades ópticas de córnea e/ou do cristalino é possível, porém de alto custo ou baixo prognóstico.

Considerando que o trauma ocular é a causa mais frequente de perda de visão na população geral e que isso acontece majoritariamente em homens em idade produtiva, em face dos achados atuais ${ }^{(19-20)}$, podemos avaliar que o efeito reabilitador da não intervenção e da reeducação para a nova condição pode ser tão ou mais produtivo em termos individuais e coletivos do que intervenções caras, sucessivas e de baixo prognóstico.

Um estudo recente, envolvendo um grupo grande de latinos ou descendentes, residentes nos Estados Unidos da América, usando o NEI-VFQ-25 mostrou que a baixa de visão está diretamente relacionada à qualidade de vida, e que diferentes doenças apresentam impacto diferente em diversos aspectos da vida cotidiana ${ }^{(21)}$. O estudo também examinou o efeito da baixa de visão monocular e observou que essa condição afetou até 10 dos 14 domínios estudados, em comparação a controles, porém, não separou casos congênitos de adquiridos.

Assim, a decisão sobre intervenções oculares, deve ser individualizada, levando em conta as características do paciente, riscos e benefícios do tratamento. Dificilmente tantas variáveis poderiam ser alocadas em algoritmos de protocolos de conduta, como existem para algumas condições sistêmicas.

É interessante notar que diante das respostas obtidas, fica a recomendação para considerar treinamentos de reabilitação à nova condição visual, individualizando as maiores dificuldades em cada caso e oferecendo treinamento específico, também, a pacientes com baixa de visão monocular. Atualmente, apenas pacientes com dificuldade binocular, são rotineiramente enviados para programas de treinamento e reabilitação.

A ferramenta usada nesse estudo, o VF-14, já foi usada anteriormente no Brasil, porém apresenta limitações ${ }^{(22-23)}$. A primeira a ser mencionada é de apurar apenas atividades diretamente relacionadas à visão e assim, não avaliar aspectos da qualidade de vida. Ficamos sem saber se a qualidade vida de pacientes cegos monoculares congênitos é similar a de pacientes com boa visão binocular. O que confrontaria estudos que advogam cirurgias de catarata bilaterais simultâneas ${ }^{(13)}$.

Outra limitação é pelo fato do questionário não ter passado por todas as etapas de validação. No nosso estudo, o VF-14 foi traduzido para o português, traduzido de volta para o inglês e essa versão manteve as características originais. Porém, a coleta de dados na população local, para avaliar a consistência das respostas, e a conclusão da validação, ainda não foram feitas, como recomendado para ferramentas usadas para qualidade de vida ${ }^{(24-25)}$. Por último, o estudo não procurou estratificar os grupos por complexidade de atividades laborais ou doenças, o que poderia indicar que em algumas delas, a visão monocular é um fator limitante.

\section{CONCLUSÃO}

A baixa de visão monocular adquirida e binocular tem impacto desfavorável na qualidade de visão, porém é significativamente mais intensa na binocular. O estudo sugere que em casos monoculares medidas terapêuticas ou de reeducação podem ter impacto similar, porém essa afirmação merece estudo específico.

\section{REFERÊNCIAS}

1. Schellini SA, Durkin SR, Hoyama E, Hirai F, Cordeiro R, Casson RJ, et al. Prevalence and causes of visual impairment in a Brazilian population: the Botucatu Eye Study. BMC Ophthalmol. 2009;9:8.

2. Salomao SR, Cinoto RW, Berezovsky A, Araújo-Filho A, Mitsuhiro MR, Mendieta L, et al. Prevalence and causes of vision impairment and blindness in older adults in Brazil: the Sao Paulo Eye Study. Ophthalmic Epidemiol. 2008;15(3):167-75.

3. Steinberg EP, Javitt JC, Sharkey PD, Zuckerman A, Legro MW, Anderson GF, et al. The content and cost of cataract surgery. Arch Ophthalmol. 1993;111(8):1041-9.

4. Moffatt SL, Cartwright VA, Stumpf TH. Centennial review of corneal transplantation. Clin Experiment Ophthalmol. 2005;33(6):642-57. Comment in: Clin Experiment Ophthalmol. 2006;34(4):387-8.

5. Maeno A, Naor J, Lee HM, Hunter WS, Rootman DS. Three decades of corneal transplantation: indications and patient characteristics. Cornea. 2000;19(1):7-11.

6. Vieira Silva J, Julio de Faria e Sousa S, Mafalda Ferrante A. Corneal transplantation in a developing country: problems associated with technology transfer from rich to poor societies. Acta Ophthalmol Scand. 2006;84(3):396-400.

7. Oliveira DF, Lira RP, Lupinacci AP, Paccola M, Arieta CE. Cataract surgery complications as a cause of visual impairment in a population aged 50 and over. Cad Saude Publica. 2008;24(10):2440-4.

8. Castells X, Alonso J, Ribo C, Casado A, Buil JA, Badia M, et al. Comparison of the results of first and second cataract eye surgery. Ophthalmology. 1999;106(4):676-82.

9. Javitt JC, Steinberg EP, Sharkey P, Schein OD, Tielsch JM, Diener M, et al. Cataract surgery in one eye or both. A billion dollar per year issue. Ophthalmology. 1995; 102(11):1583-92; discussion 1592-3.

10. Dandona L, Naduvilath TJ, Janarthanan M, Ragu K, Rao GN. Survival analysis and visual outcome in a large series of corneal transplants in India. Br J Ophthalmol. 1997:81(9):726-31.

11. Norregaard JC, Bernth-Petersen P, Alonso J, Dunn E, Black C, Andersen TF, et al. Variation in indications for cataract surgery in the United States, Denmark, Canada, and Spain: results from the International Cataract Surgery Outcomes Study. Br J Ophthalmol. 1998;82(10):1107-11. Comment in: Br J Ophthalmol. 1998; 82(10):1101-2.

12. Arshinoff SA, Strube YN, Yagev R. Simultaneous bilateral cataract surgery. J Cataract Refract Surg. 2003;29(7):1281-91. Comment in: J Cataract Refract Surg. 2004.30(8):1610-1.

13. Lundstrom M, Albrecht S, Nilsson M, Astrom B. Benefit to patients of bilateral same- 
day cataract extraction: Randomized clinical study. J Cataract Refract Surg. 2006; 32(5):826-30

14. Associação Brasileira dos Deficientes com Visão Monocular. Memorial Portadores de visão monocular-ABDVM [Internet]. Disponível em: http://www.visaomonocular.org/ documentos.asp

15. Sampaio R, Held E, Cohen EJ, Grewald S, Rapuano CJ, Smith SF, et al. Binocular vision recovery in bilateral keratoplasty. Cornea. 2001:20(5):471-4

16. Castells X, Comas M, Alonso J, Espallargues M, Martinez V, García-Armi J, et al. In a randomized controlled trial, cataract surgery in both eyes increased benefits compared to surgery in one eye only. J Clin Epidemiol. 2006:59(2):201-7.

17. Avakian A, Temporini ER, Kara-Jose N. Second eye cataract surgery: perceptions of a population assisted at a university hospital. Clinics (Sao Paulo). 2005:60(5):401-6.

18. Steinberg EP, Tielsch JM, Schein OD, Javitt JC, Sharkey P, Cassard SD, et al. The VF14. An index of functional impairment in patients with cataract. Arch Ophthalmol. 1994:112(5):630-8.

19. Cecchetti DF, Cecchetti SA, Nardy AC, Carvalho SC, Rodrigues M de L, Rocha EM A clinical and epidemiological profile of ocular emergencies in a reference emergency center]. Arq Bras Oftalmol. 2008;71(5):635-8. Portuquese.
20. May DR, Kuhn FP, Morris RE, Witherspoon CD, Danis RP, Matthews GP, et al. The epidemiology of serious eye injuries from the United States Eye Injury Registry. Graefes Arch Clin Exp Ophthalmol. 2000;238(2):153-7.

21. Broman AT, Munoz B, Rodriguez J, Sanchez R, Quigley HA, Klein R, et al. The impact of visual impairment and eye disease on vision-related quality of life in a Mexican-American population: proyecto VER. Invest Ophthalmol Vis Sci. 2002; 43(11):3393-8

22. Cavalcanti MT, Mahon M, Nóbrega DAT, Remígio MC, Pires CS. Ceratocone: resultados visuais, complicações e qualidade de vida após ceratoplastia penetrante realizada por médico residente. Arq Bras Oftalmol . 2004:67(3):415-8.

23. Atique D, Goulart DG, Lake JC, Lima FA, Felberg S, Nishiwaki-Dantas MC. Qualidade de vida após transplante penetrante de córnea. Arq Bras Oftalmol. 2002;65(3):351-4

24. Ferreira E, Dantas RA, Rossi LA, Ciol MA. The cultural adaptation and validation of the "Burn Specific Health Scale-Revised" (BSHS-R): version for Brazilian burn victims. Burns. 2008:34(7):994-1001

25. Patient-Reported Outcome and Quality of Life Instruments Database - PROQOLID [computer program]. Lyon, France: Mapi Research Institute; 2010. Available from: http://www.proqolid.org/

\title{
50 Congresso da Sociedade Brasileira de Lentes de Contato, Córnea e Refratometria-SOBLECO
}

\author{
15 a 17 de abril de 2011 \\ Estação Embratel Convention Center \\ Curitiba - PR
}

Informações:

Creative Solution

Tels.: (11) 5575-0254 e 5539-2186

Site: www.creativesolution.com.br 